\title{
Non-contact measurement of displacer motion of a miniature split-Stirling cryocooler
}

\author{
Yee-Pien Yang *, Hsin-Tang Chien, June-Ming Chen \\ Department of Mechanical Engineering, National Taiwan University, Taipei, Taiwan 106, ROC
}

\begin{abstract}
A relative displacement measurement method is originally proposed to calibrate the static sensitivity of the measurement of displacer motion inside the cold finger of a split-type Stirling cryocooler. This measurement is non-contact and nondestructive by the use of the well-known linear variable-differential transformer (LVDT). Therefore the cooler operates in normal conditions without any interference, such as loading effects, from the measurement. The true displacement of the displacer is the product of the output voltage from the LVDT and the calibrated static sensitivity. Since the displacer motion consists of multiple sinusoidal signals and its oscillation center drifts continuously, modifications are made to get a better estimation of the static sensitivity. Both the direct fitting method and the small drift modification method are investigated through computer simulations, and are used successfully in experiments on a Hughes $7013 \mathrm{H}$ cooler.
\end{abstract}

Keywords: Split-Stirling cryocooler; Static sensitivity; Relative displacement measurement method; Direct curve fitting; Small drift modification

\section{Introduction}

Split-type Stirling cryocoolers are characterized by complete separation of the expansion cylinder from the compressor cylinder, drive motor and crankcase. These cryocoolers have been made small, lightweight, low power and more efficient in terms of their coefficient of performance (COP) to provide a cryogenic environment for cold electronics. The fine-bore gasfilled metal tube that connects the expansion and compression cylinders is sufficiently malleable. This not only permits the low-mass component to be mounted in such applications as ultra-sensitive magnetometers and gigaflop-speed supercomputers, but also results in the isolation of cold electronic systems from environment noise, compressor-induced vibration and elec-

\footnotetext{
* Corresponding author.
}

tromagnetic emissions from the compressor and the motor. Therefore, the displacer in the expansion cylinder is free and oscillates simply as a result of the gas force acting on it. The gas force is provided by the actuating piston oscillating in the compression cylinder with a phase shift relative to the displacer motion. The stroke variation of the displacer indicates the volume change in the expansion space, and its pressurevolume diagram illustrates the work done by the gas on the displacer. The net area of the diagram will represent the heat transferred to the expansion space, a positive work, so there is some refrigeration effect, and the tip of the cold finger will become cold.

In the design of a split-type miniature Stirling cryocooler, the displacer motion is closely related to the available refrigeration [1], which depends on the cooling or ambient atmospheric temperature, working fluid pressure, the swept volume in the compression space 
or the expansion space, operating frequency, etc. Under a certain operating pressure and temperature, the available refrigeration depends on the swept volume, or on the stroke of the displacer per unit refrigeration cycle. The principal problems with split-Stirling cryocoolers are usually to maintain a proper stroke of the reciprocating displacer in the expander cylinder, and to regulate its phase relationship with the motion of the compressor piston. In fact, the displacer stroke varies under different operation conditions, such as gas pressure drop, input power, compressing frequency, mass flow-rate, ambient temperature, heat loss, mechanical friction loss, etc. Most of these operating characteristics are specified by analysis and simulation so as to ensure satisfactory displacer stroke and hence available refrigeration. The measurement of the displacer's displacement becomes essential during the development of split-type Stirling cryocoolers, from the prototype testing to the quality control process of the product.

The measurement of the displacer motion is important in the identification of dynamical properties of split-Stirling cryocoolers. Xiang et al. [4] took the displacer motion signal to identify the moving damping of the displacer, which changes along with the lowering of the refrigeration temperature, in a split cycle free piston Stirling cryocooler. Zhang et al. [5] conducted an experimental investigation on the dynamic pressure distribution in a split piston Stirling cryocooler system, where the displacer motion was measured with a Halleffect displacement transducer. Stolfi and Jonge [6] regulated the motions of the displacer and the piston of a Briarcliff cryocooler by feeding their displacement signals back into a control system to adjust the current in the linear motors. Capacitive position pickups were also used to measure both the compressor piston and displacer motions in BAe $20 \mathrm{~K}$ to $80 \mathrm{~K}$ split-Stirling coolers for the control system to maintain the required phase relationship between the compressor and displacer [7]. The above measurement methods rely on built-in sensors installed inside the system of a closed chamber.

One might raise a question: could we measure the displacement of the displacer inside the cold finger with neither built-in sensors nor sensors directly contacting the displacer? Built-in sensors increase the cost and complexity in the product design and manufacture, and are redundant when control on the dis- placer motion is not required. Besides, the contacttype sensors usually cause loading effects on the measured medium. This paper proposes an alternative noncontact measurement technique for measuring the displacer motion. Neither installation of sensors inside the cooler nor destruction of the cylinder wall is required as the measurement is performed. The objective of such measurement is to identify the dynamical characteristics of a cryocooler during its development from the design, manufacture through the quality control process of the product.

\section{Measurement method and difficulties}

Linear variable-differential transformers (LVDTs) are used to measure displacement by the principle of electromagnetic induction, and are termed as variableinductance transducers. The basic arrangement of the LVDT constitutes a primary winding and a secondary winding, symmetrically wound in the two end segments. The primary coil carries an $\mathrm{AC}$ excitation that induces a steady $\mathrm{AC}$ voltage in the two secondary coils connected in series opposition. The level of the induced voltage depends on the flux linkage between coils. The reluctance of the flux path varies with the position of the iron core due to its relative permeability, which indicates how a magnetic material changes the magnetic induction compared with the induction that would be observed in free space. The iron core is connected to the object whose position is to be measured, the voltage induced in the two secondary coils varying with the position of the iron core. This core is usually included in commercially available LVDTs, and is made of ferromagnetic material inserted coaxially into the cylindrical, insulating, nonmagnetic form without actually touching it. With built-in microelectronics or some read-out systems for signal conditioning, either by the rectification or by the demodulation method [2], the output is directly proportional, in the linear operating range, to the displacement.

In this paper, a different way of using a LVDT in measuring the position of the displacer is developed. The LVDT housing containing the coils is mounted outside the cold finger cylinder, inside which the displacer now plays the role of the commercial iron core. The cylinder wall is usually made of 300 series stainless steel with low permeability. Displacers are nor- 
mally equipped with regenerator matrix composed of any finely divided material $[2,9]$.

Difficulties in measuring the displacement occur due to the following facts:

(i) The regenerator matrix material may have very low magnetic permeability compared with ferromagnetic materials.

(ii) The cylinder wall thickness of the cold finger produces a shielding effect on the induced change of the flux linkage in the secondary coils.

Both these facts result in the attenuation of the induced voltage in the secondary coils. The output signal, therefore, must be calibrated to identify the input signal-the true displacement of the displacer motion. When an input-output calibration has been performed, the static sensitivity $K$ can be defined as the slope of the calibration curve [3], i.e.,

$K=\frac{\Delta y}{\Delta v}$,

as an indication of the change of true displacement $\Delta y$ per unit voltage output $\Delta v$. It is apparent that $K$ is a function of the magnetic permeability of the regenerator matrix, cylinder wall thickness, ambient temperature, operating frequency of the Stirling cryocooler, etc.

\section{Determination of static sensitivity-Relative displacement measurement method}

The true displacement of the displacer can be obtained by multiplying the LVDT output voltage with the static sensitivity $K$. To calibrate the static sensitivity, the relative displacement measurement method is proposed. First, we assume that the displacer oscillates with a single sine function. The stroke, or the maximum displacement, of the displacer will be the distance of two vertical positions of the LVDT, i.e., the lowest and the highest measurement locations. The former is where the maximum voltage output, reading the peak value of the sinusoidal signal, is zero; while the latter is where the minimum voltage output is zero. The position of the LVDT is measured by an optical displacement sensor isolated from the cryocooler with respect to a pre-specified reference. We did not use a dial gauge because the optical sensor provided much better accuracy. Then, the static sensitivity becomes the quotient of half the stroke divided by the amplitude of the voltage output.

Theory. In measuring the displacer motion inside a closed cylinder, we assume

(i) the displacer oscillates in a steady-state motion of a single sine wave,

(ii) the center of the displacer motion is fixed, and

(iii) there is no measurement noise.

The static sensitivity of the measurement is calibrated by

$K=\frac{\left|y_{\max }-y_{\min }\right|}{2 A}$,

in which $y_{\min }$ is the lowest measurement location of the LVDT where its minimum output voltage is zero, $y_{\max }$ is the highest measurement location of the LVDT where its maximum output voltage is zero, and $A$ is the amplitude of the sine wave voltage output.

Proof. The output voltage of the LVDT at the position in the experimental apparatus shown in Fig. 1 is

$v(y, t)=\frac{1}{K}[D \sin \omega t+y]$,

where $D$ is the actual amplitude of the displacer oscillating at frequency $\omega$, and $y$ is the distance between the oscillation center of the displacer and the null position of the LVDT. Let the center of displacer motion be the origin of the $y$-axis and its positive direction go downward.

The lowest measurement point is located where the null coincides with the lower peak of the displacer motion, and the output voltage becomes

$v\left(y_{\min }, t\right)=\frac{1}{K}\left[D \sin \omega t+y_{\min }\right]$

and

$\min \left[v\left(y_{\min }, t\right)\right]=\frac{1}{K}\left[-D+y_{\min }\right]=0$.

Similarly, at the highest measurement point,

$\max \left[v\left(y_{\max }, t\right)\right]=\frac{1}{K}\left[D+y_{\max }\right]=0$.

These yield

$K=\frac{D}{A}=\frac{\left|y_{\max }-y_{\min }\right|}{2 A}$. 
By definition $K$ is the slope of the straight line describing the true displacement per output voltage if the measurement is performed in the linear range of sensors and the measured signal is not contaminated with noise. From a statistics point of view, a sufficient number of measurements is necessary to get an accurate estimate of $K$ by the least-squares fit for a linear curve [3]. Therefore, it is not necessary to locate the LVDT at the lowest and highest measurement points, but the range of measurement locations spanned must be large enough. Then, the actual amplitude of the displacer oscillation can be easily determined by the interpolation method described in what follows.

\subsection{Experiment I-Verification of relative displacement measurement method}

To simulate the sinusoidal motion of the displacer, we set up an experiment as shown in Fig. 1. An eccentric wheel of radius $10 \mathrm{~mm}$ driven by a DC servo motor rotates in a block to which a displacer made of iron is connected through a rod made of stainless steel. The amplitude of the sinusoidal motion produced by the eccentric wheel is its eccentricity, $2.25 \mathrm{~mm}$, and the stroke is two times the eccentricity. The ratio of radius and eccentricity as well as the roundness of

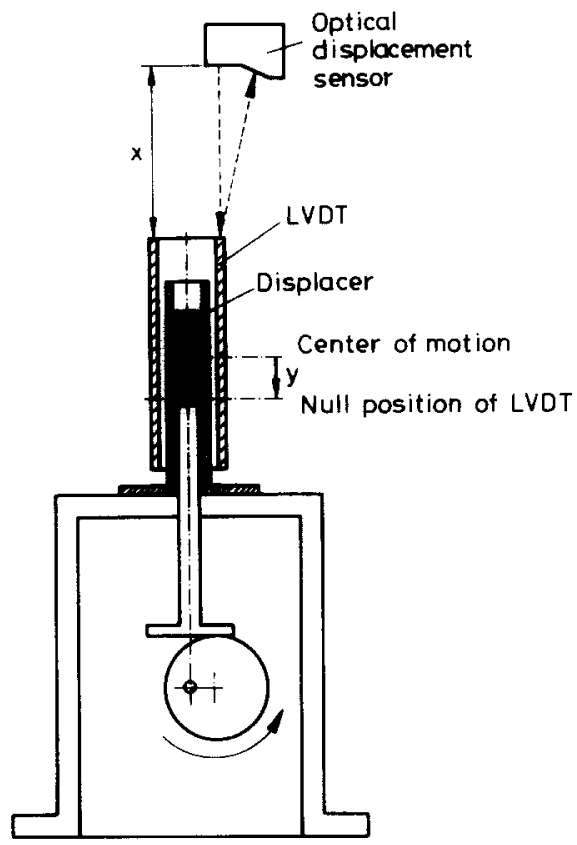

Fig. 1. Measurement apparatus of experiment I.
Table 1

Technical data of LVDT and optical displacement sensor

\begin{tabular}{ll}
\hline & Specifications \\
\hline LVDT & \\
Model & MTN/E-010, Monitran Ltd. \\
Range & $\pm 10 \mathrm{~mm}$ \\
Body length & $73 \mathrm{~mm}$ \\
Sensitivity & $140 \mathrm{mV} / \mathrm{V} \mathrm{FRO} \mathrm{at} 3 \mathrm{kHz}$ \\
Excitation & $5 \vee \mathrm{RMS}$ at $3 \mathrm{kHz}$ \\
AC null & $<10 \mathrm{mV}$ \\
Linearity & $<0.5 \%$ \\
Optical displacement sensor & \\
Model & PA 1830, Keyence Corp. \\
Reference distance & $40 \mathrm{~mm} \pm 1 \mathrm{~mm}$ \\
Measurement range & $\pm 5 \mathrm{~mm}$ \\
Resolution & $10 \mu \mathrm{m}$ \\
Accuarcy & $\pm 20 \mu \mathrm{m} \pm 1 \%$ \\
& of measured value \\
\hline
\end{tabular}

$33.62 \mu \mathrm{m}$ of the eccentric wheel yield a smooth sine wave motion of the displacer in this experiment. The frequency of the displacer oscillation is $30 \mathrm{~Hz}$ tuned by the input voltage of the DC motor. The sampling rate is $300 \mathrm{~Hz}$.

At each measurement location, the sample function of the voltage output was measured by the LVDT, while the location of the LVDT was recorded by the optical displacement sensor. The technical data of these sensors are listed in Table 1. Since the sample function of voltage output of the LVDT is corrupted with measurement noise, its mean values of lower and upper peaks are obtained by their time averages. The real lines in Fig. 2(a) show the minimum and maximum voltages versus the position of the LVDT, and these lines are fitted by the least-squares algorithm. Since the purpose of this experiment was to verify the proposed measurement method, the displacer was made of iron of maximum relative permeability 5000 [8], so as to ensure the sensitivity of the LVDT provided by the OEM manufacturer. The stroke is estimated by the distance of two points where the maximum and minimum voltage lines intersect with the zero-voltage line, which is $4.535 \mathrm{~mm}$ with a confidence of $99.007 \%$. These two intersections are, respectively, the lowest and the highest measurement locations. The corresponding static sensitivity of the measurement is $16.59 \mathrm{~mm} / \mathrm{V}$ which is the slope of the mean voltage line in the figure. 

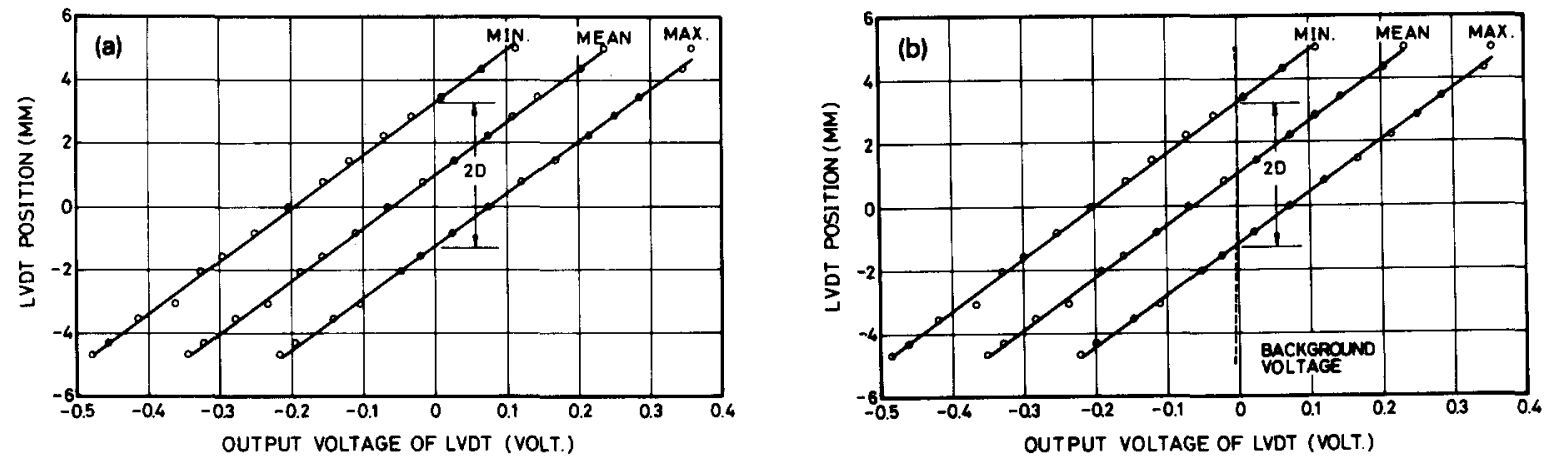

Fig. 2. Determination of static sensitivity and displacer stroke (2D). (a) Without background voltage compensation, true static sensitivity $=16.59 \mathrm{~mm} / \mathrm{V}, 2 D=4.535 \mathrm{~mm}, 99.007 \%$ confidence; (b) with background voltage compensation, static sensitivity $=16.45 \mathrm{~mm} / \mathrm{V}, 2 D=4.497 \mathrm{~mm}, 99.842 \%$ confidence. True static sensitivity is $4.50 \mathrm{~mm}$.

\subsection{Shielding effect of cylinder wall}

In the above experiment and analysis, we assume that the null voltage of the LVDT surrounding the cylinder corresponds to identical relative positions of the core to the LVDT coils. The null voltage coinciding with the original null specified by the LVDT manufacturer is in the center of the measuring range. Therefore, the output voltage in the above experiment keeps a constant bias from the original null voltage measured if the cylinder were absent. This constant bias on the output voltage will not change the result of the static sensitivity, because the slope of the minimum/maximum output voltage against the LVDT location is independent of the null reference. However, the length of the cylinder and the LVDT body are finite, resulting in uneven and different distributions of the magnetic flux path at various measurement locations of LVDT. Thus, the null voltage, namely the background voltage, depends on the measurement location of the LVDT. The dashed line in Fig. 2(b) shows the background voltage of the LVDT surrounding the cylinder at different locations. In calibrating the background voltage, the displacer was removed from our experimental set-up. The slope of the mean voltage line in Fig. 2(b) is $16.45 \mathrm{~mm} / \mathrm{V}$ as an improved estimate of $K$ with background voltage compensation. Also estimated is the stroke of $4.497 \mathrm{~mm}$ with a confidence of $99.842 \%$, which is less than that estimated without background voltage compensation.

In measuring the displacer motion of a working Stirling cryocooler, however, one may neglect the shield- ing effect of the cylinder wall owing to the following reasons. First, the cylinder wall of the cold finger is usually made long and thin to minimize thermal conduction from the warm space (the bottom part of the cold finger) to the cold region (the expansion chamber). Second, the thin wall that is usually made of stainless steel to meet the high strength requirement is of low magnetism. The thin thickness and low magnetic characteristics of the cylinder wall yield a negligible distortion of the magnetic flux distribution in the measurement space surrounded by LVDT. Hence, the background voltage can be regarded as a constant bias over the measurement region.

\section{Displacer motion with multiple sine components}

The displacer motion from a typical test on a small Hughes $7013 \mathrm{H}$ split-Stirling cryocooler is a combination of multiple sinusoidal signals, as shown in Fig. 3(a). Its power spectrum density function, Fig. 3(b), shows that the signal consists of a primary frequency content around $30 \mathrm{~Hz}$, a low-frequency carrier and several high-frequency components. The energy of the primary frequency component being much larger than that of the remaining frequency components motivates the following modification of determining the static sensitivity. Let the displacement of the displacer be expressed as

$d(y, t)=y+D \sin \omega t+\sum_{i=1}^{n} D_{i} \sin \left(\omega_{i} t+\phi_{i}\right)$, 
where $y$ is the distance from the oscillation center of the displacer to the null position of the LVDT, $D$ is the amplitude of the major sine wave motion of frequency $\omega$, each $D_{i}$ denotes the amplitude of a minor sine wave motion of frequency $\omega_{i}$ and phase $\phi_{i}$. The output voltage of the LVDT becomes

$v(y, t)=\frac{1}{K} d(y, t)+\nu(y)+\eta(t)$,

where $\nu(y)$ is the null voltage of the LVDT surrounding the cold finger, and $\eta(t)$ is the measurement noise which is assumed to be a white Gaussian random process with zero mean. Taking the time average of $v(y, t)$ by $N$ samples along a sample function and letting $v(y)$ be the expectation value of $v(y, t)$, we have

$$
\begin{aligned}
v(y)=\mathcal{E}[v(y, t)] & \\
=\frac{1}{K} & \left\{y+\frac{1}{N} \sum_{j=1}^{N}\left[D \sin \omega t_{j}\right.\right. \\
& \left.\left.+\sum_{i=1}^{n} D_{i} \sin \left(\omega_{i} t_{j}+\phi_{i}\right)\right]\right\}+\nu(y) .
\end{aligned}
$$

It is easy to verify that

$$
\sum_{j=1}^{N}\left[D \sin \omega t_{j}+\sum_{i=1}^{n} D_{i} \sin \left(\omega_{i} t_{j}+\phi_{i}\right)\right]=0
$$

if

$$
N-1=\operatorname{LCM}\left(\frac{1}{f \Delta t}, \frac{1}{f_{i} \Delta t}, \ldots, \frac{1}{f_{n} \Delta t}\right),
$$

where $f=\omega / 2 \pi, \Delta t=t_{i}-t_{i-1}$, and Eq. (10) becomes

$v(y)=\frac{1}{K} y+\nu(y)$.

From the discussion of the last section, the null voltage of the LVDT is usually small enough to be neglected. The static sensitivity can still be determined by Eq. (1) provided that the length of the sample function satisfies the condition of Eq. (12). However, the procedure of selecting a proper length for a sample function is tedious and impractical.

\subsection{Experiment II-Direct curve fitting}

To relieve the restriction of (12) in determining the static sensitivity of the measurement, we propose the
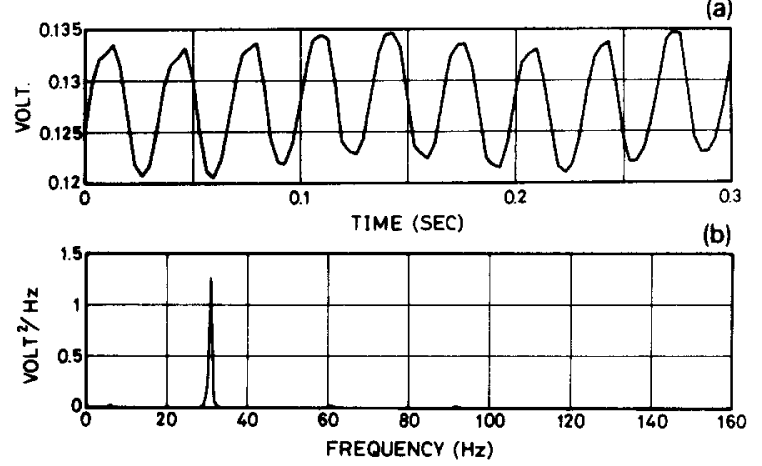

Fig. 3. (a) LVDT output without filtering; (b) Power spectrum of (a).

following modification in the processing of the experimental data. Since the primary sinusoidal component of the output voltage possesses the majority of energy over the remaining components, and all the components separate in frequency apparently, it is easy to use a bandpass filter to remove minor components without attenuating the major one. Therefore, the same procedure to determine the static sensitivity for a single sine signal is performed. We did not use digital Fourier transforms because we need time history records in the following procedures determining the static sensitivity of the measurement:

(i) Record a sample function of the displacer motion in terms of output voltages of the LVDT located at $y_{i}$ that are measured by an optical displacement sensor, as shown in Fig. 3.

(ii) Use a digital bandpass filter to retain the primary sine-wave component of the sample function as shown in Fig. 4.

(iii) Calculate the time-average of the mean and peak (maximum and minimum) values (in volts) of the filtered sample function.

(iv) Repeat (i) through (iii) at various measurement locations of the LVDT, and plot the mean/peak voltages against the measurement locations.

(v) Fit the data with straight lines.

The slope of the line fitting the mean value of each sample function is chosen to be the best estimate of the static sensitivity of the measurement. The primary stroke, or the stroke of the primary sine-wave component, is estimated by the distance of the two intersections at which the maximum voltage curve, the minimum voltage curve and the zero voltage line intersect. 

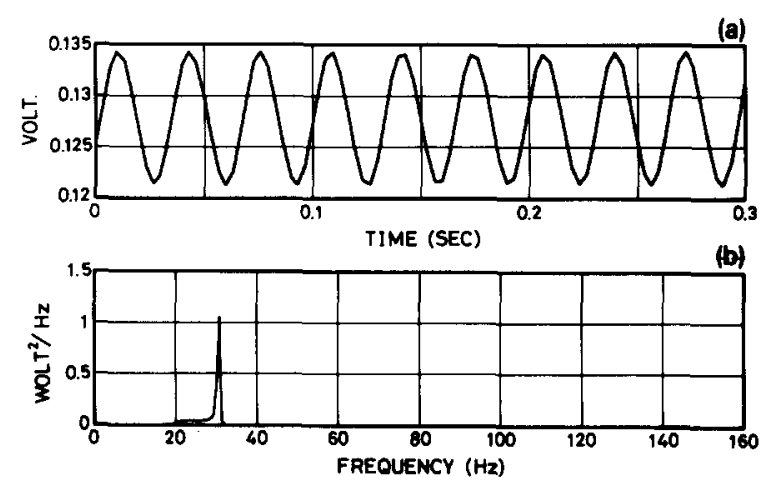

Fig. 4. (a) Filtered LVDT output of Fig. 3(a); (b) Power spectrum of (a).

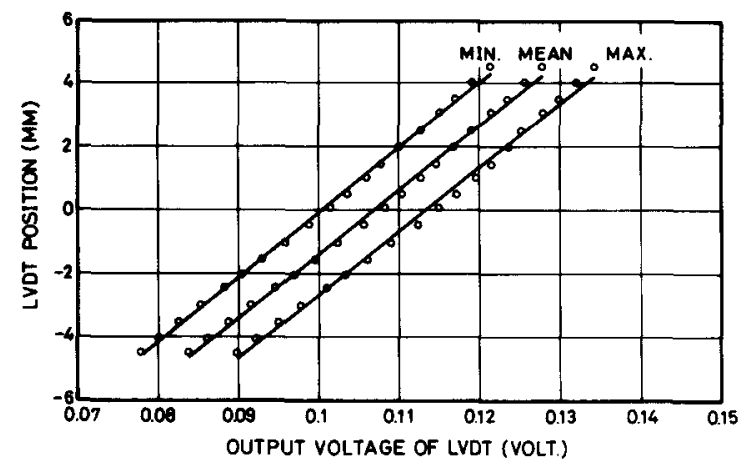

Fig. 5. Experiment on the static sensitivity estimate of $202.9 \mathrm{~mm} / \mathrm{V}$, the estimated stroke of the primary sine wave is $2.63 \mathrm{~mm}$, direct fitting method.

This procedure is called the direct fitting method to determine the static sensitivity of the measurement of the displacer motion inside a closed cylinder.

Fig. 5 shows the result of the static sensitivity of the measurement of displacer motion of a Hughes $7013 \mathrm{H}$ split-Stirling cryocooler. An eighth-order digital bandpass Butterworth filter of bandwidth $20-40 \mathrm{~Hz}$ was used to filter out minor sine wave components in each sample function. The estimated static sensitivity is $202.9 \mathrm{~mm} / \mathrm{V}$ and the average stroke estimate of the primary sine wave is $2.63 \mathrm{~mm}$ by averaging strokes at all LVDT locations.

\section{Displacer drift}

In the direct fitting method of determining the static sensitivity, we assumed that the free displacer motion had a consistent center of oscillation. This may not be true for the displacer motion over a long time period even in the steady state. It was found that the oscillation center of the displacer motion drifted. This drift may depend on various operating conditionsambient temperature, cold temperature, gas pressure, heat load, heat loss, etc. To determine the static sensitivity, the amount of the displacer drift must be extracted from $y$, the position of the center of the displacer motion, as if no drift had happened. However, the experimental set-up does not allow us to measure the displacer motion and its drift simultaneously.

Proposed is a modified estimation method for static sensitivity instead of direct curve fitting of the output voltage against the measurement location of the LVDT. Since the LVDT is moved along the cylinder when the displacer motion is measured, the drift of its oscillation center varies during the measurement. The output voltage will be expressed by

$v(y, t)=\frac{1}{K}[d(y, t)-\delta(y, t)]+\nu(y)+\eta(t)$,

where $\delta(y, t)$ presents the drift. By taking the expectation of $v(y, t)$ and neglecting small background null voltages, Eq. (14) can be expressed at each measurement point $j$ as follows:

$v_{j}=\frac{1}{K}\left(y_{j}-\delta_{j}\right), \quad j=0,1,2, \ldots, m$,

where $\delta_{0}$ is set zero as a reference datum for the other $m$ measurement points. Then, we have

$$
\begin{aligned}
\Delta y_{j} & \stackrel{\Delta}{=} y_{j}-y_{0} \\
& =K\left(v_{j}-v_{0}\right)+\delta_{j}, \quad j=1,2, \ldots, m .
\end{aligned}
$$

Since the drift varies slowly, one may assume that the difference in drifts at two adjacent measurement points is small compared to the stroke of the displacer, i.e.,

$\left|\delta_{j+1}-\delta_{j}\right| \ll \epsilon \ll 1, \quad \epsilon>0$.

This gives rise to the name of small drift modification method for the estimation of the static sensitivity. Therefore,

$$
\begin{aligned}
\Delta y_{j+1}-\Delta y_{j} \cong K\left(v_{j+1}-v_{j}\right) & \\
j & =1,2, \ldots, m-1 .
\end{aligned}
$$


The combination of Eqs. (16) and (18) yields $m-1$ matrix equations

$M_{j} \Delta y=N_{j} \theta, \quad j=1,2, \ldots, m-1$,

where

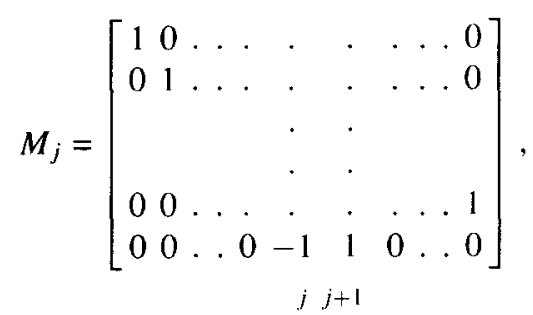

$N_{j}=\left[\begin{array}{ccccc}1 & 0 & \ldots & 0 & v_{1}-v_{0} \\ 0 & 1 & \ldots & 0 & v_{2}-v_{0} \\ & \vdots & & \vdots \\ 0 & 0 & \ldots & 1 & v_{m}-v_{0} \\ 0 & 0 & \ldots & 0 & v_{j+1}-v_{j}\end{array}\right]$,

$\Delta y=\left[\begin{array}{c}\Delta y_{1} \\ \Delta y_{2} \\ \vdots \\ \Delta y_{m}\end{array}\right], \quad \theta=\left[\begin{array}{c}\delta_{1} \\ \delta_{2} \\ \vdots \\ \delta_{m} \\ K\end{array}\right]$.

For the $j$ th equation of (19), an estimate of $\theta$ can be obtained by

$\hat{\theta}_{j}=\left[\begin{array}{lllll}\hat{\delta}_{1} & \hat{\delta}_{2} & \cdots & \hat{\delta}_{m} & \hat{K}\end{array}\right]^{\mathrm{T}}=N_{j}^{-1} M_{j} \Delta y$,

where $N_{j}$ is invertible if and only if $\left(v_{j+1}-v_{j}\right)$ is not zero.

For some estimates $\hat{\theta}_{k}$, the resulting $\left|\hat{\delta}_{k+1}-\hat{\delta}_{k}\right|$ may be larger than the prespecified index $\epsilon$, violating the assumption Eq. (17). Therefore, the estimate of $\theta$, and hence $K$, will be obtained by the unbiased average

$\hat{\theta}=\frac{1}{m-1} \sum_{j=1}^{m-1} \alpha \hat{\theta}_{j}$,

where

$\alpha= \begin{cases}1 & \text { if }\left|\hat{\delta}_{k+1}-\hat{\delta}_{k}\right|<\epsilon, \\ 0 & \text { otherwise }\end{cases}$

\subsection{Simulation}

To validate the modified method of estimating the static sensitivity, we simulated the drift phenomenon

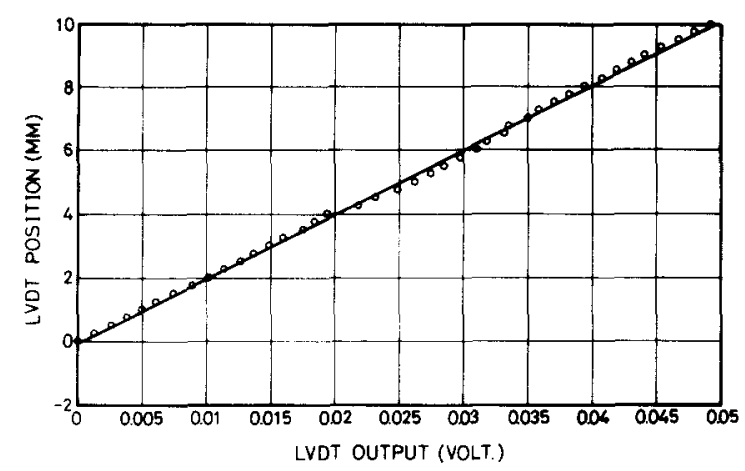

Fig. 6. Simulation of the static sensitivity estimate, direct fitting method (static sensitivity estimate: $203.1 \mathrm{~mm} / \mathrm{V}$, actual static sensitivity: $200 \mathrm{~mm} / \mathrm{V}$, confidence: $98.458 \%$ ).

by computer programs, in which a proper static sensitivity $K(=200 \mathrm{~mm} / \mathrm{V})$ and the drift history $\delta_{j}$ were assigned. For equally spaced measurement locations $y_{j}$, the mean voltage of the LVDT output was simulated by Eq. (14). Both the direct fitting method and the small drift modification method were performed, and the static sensitivity $K$ and the corresponding drift were both calculated.

As described in the above section, for the direct fitting method, the $K$ estimate is the slope of the straight line to fit the measurement data regardless of the drift, which is $203.1 \mathrm{~mm} / \mathrm{V}$ with a confidence of $98.459 \%$ as shown in Fig. 6. Substituting this estimated $K$ into Eq. (15) with the zero reference data $\delta_{0}$ results in the drift estimate for the direct fitting method, as the solid line shown in Fig. 7. Alternatively, the drift estimate from Eq. (24) for the modified method is the dashed line in Fig. 7, where the solid line with star symbols

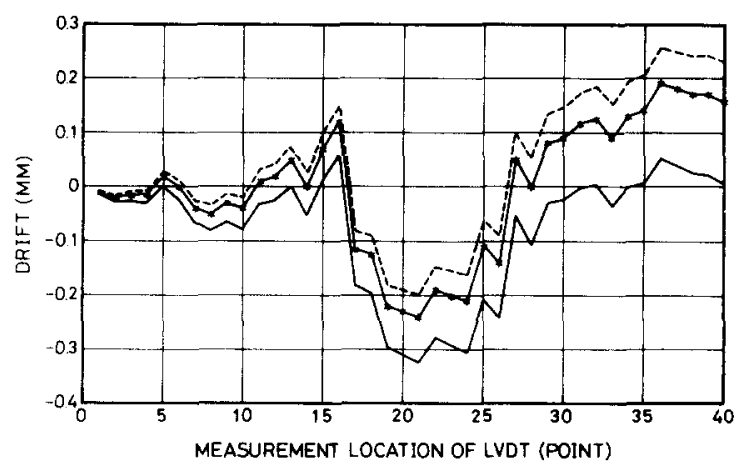

Fig. 7. Simulation of the drift estimate (dashed line: small drift modification method; solid line: direct fitting method; solid line with stars: actual drift). 


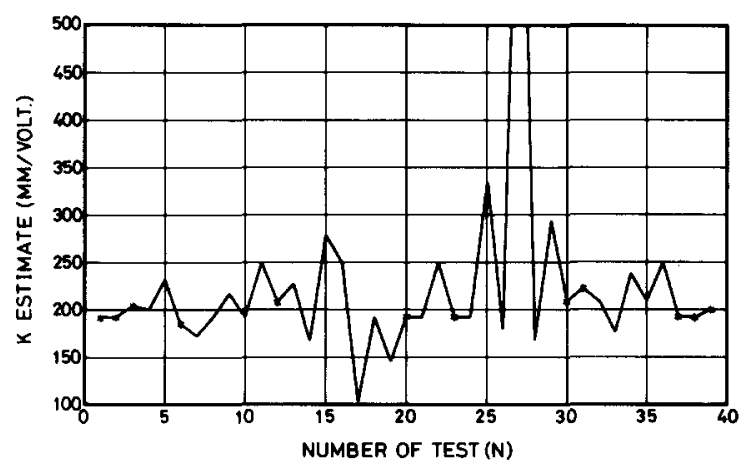

Fig. 8. Simulation of the static sensitivity estimate, small drift modification method (static sensitivity estimate: $198.5 \mathrm{~mm} / \mathrm{V}$, actual static sensitivity: $200 \mathrm{~mm} / \mathrm{V}$, confidence: $99.251 \%, \epsilon=0.05 \mathrm{~mm}$, *: data taken for estimating $K$ ).

expresses the assigned drift history. At the same time, the static sensitivity is estimated to be $198.5 \mathrm{~mm} / \mathrm{V}$ with a confidence of $99.251 \%$ as shown in Fig. 8, where stars represent the values selected to calculate $\hat{K}$ in Eq. (24) for $\epsilon=0.05 \mathrm{~mm}$, about $2 \%$ less than the actual stroke of the displacer. Note that the error in estimating the static sensitivity for the small drift modification method is not always less than that for the direct fitting method, like the case presented here, but both errors are limited and acceptable. In fact, the estimation error increases as the drift amplitude becomes larger.

\subsection{Experiment III-Small drift modification}

In this experiment, sample functions were collected at the measurement points at which the displacer drift

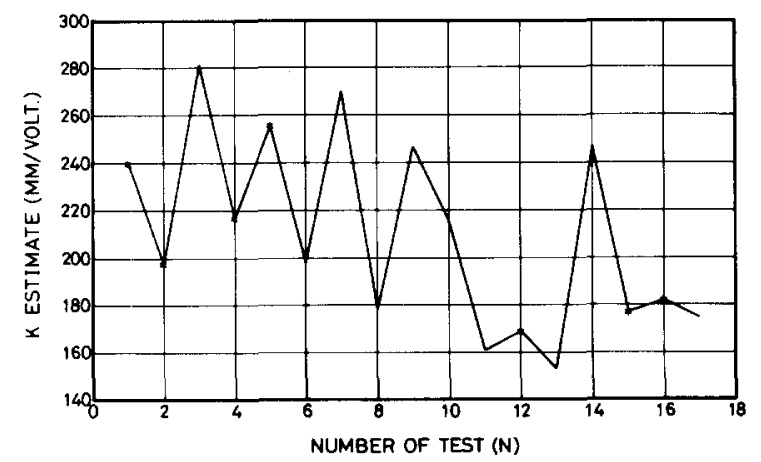

Fig. 9. Experiment on the static sensitivity estimate, small drift modification method (static sensitivity estimate: $205.2 \mathrm{~mm} / \mathrm{V}$, $\epsilon=0.05 \mathrm{~mm}$, *: data taken for estimating $K$ ).
Table 2

Static sensitivity estimate

\begin{tabular}{lll}
\hline & \multicolumn{2}{l}{ Estimate of static sensitivity $K(\mathrm{~mm} / \mathrm{V})$} \\
\cline { 2 - 3 } $\begin{array}{l}\text { Test } \\
\text { No. }\end{array}$ & $\begin{array}{l}\text { Direct fitting } \\
\text { method }\end{array}$ & $\begin{array}{l}\text { Small drift } \\
\text { modification method }\end{array}$ \\
\hline 1 & 202.9 & 205.2 \\
2 & 199.4 & 195.3 \\
3 & 216.0 & 206.3 \\
4 & 215.5 & 223.7 \\
5 & 227.8 & 226.9 \\
6 & 204.8 & 227.4 \\
7 & 212.5 & 205.4 \\
Average & 211.3 & 212.9 \\
\hline
\end{tabular}

was varying. Without violating the small drift assumption, selected points are used to calculate the estimate of $K$ via Eq. (24). Fig. 9 shows one of the experimental results. Additional estimates of static sensitivity by the use of the direct fitting method and small drift modification method are listed in Table 2, where we found that the average estimates of $K$ were very close to each other. From a statistics point of view, both methods are applicable to the estimation of static sensitivity of the displacer motion measurement.

One should be reminded that the drift was not included in the calculation of the static sensitivity for the direct fitting method, i.e., the estimation error bound was determined by the amount of drift. For large drift deviation, one would have no way to improve the estimation of static sensitivity. However, an additional criterion (17) was included in the estimation of static sensitivity. The $\epsilon$ can be selected small enough to discard the drift estimates that violate criterion (17), and those left in Eq. (24) must result in a better estimate of $K$.

\section{Summary and conclusion}

This paper has proposed a non-contact method of measuring the displacement of the displacer enclosed in the cold finger of a split-Stirling cryocooler without any built-in sensors. A calibration was performed for the static sensitivity by which the output voltage of LVDT is multiplied to obtain the true displacement of the displacer. This method, called the relative 
displacement measurement method, has been proved theoretically and validated by experiments where the displacer motion was simulated as a single sinusoidal oscillation.

In the normal operation of a miniature split-Stirling cryocooler, the displacer motion consists of multiple sine wave contents and its oscillation center drifts. Two modified methods are then proposed to estimate the static sensitivity - the direct fitting method and the small drift modification method. The former uses a digital bandpass filter to retain the primary frequency component and follows the relative displacement measurement procedures to estimate the static sensitivity. The displacer drift is ignored in this method, and no modification can be done in the estimation if the drift is large compared with the displacer stroke. The latter computes the static sensitivity and the drift of each measurement location of the LVDT simultaneously, where the criterion of a small drift difference of adjacent measurement locations is incorporated. Better estimation may be obtained by the use of the small drift modification method if the index of the criterion is small enough.

One of the limitations of the proposed non-contact measurement method is that the material of the displacer must be magnetic. For those displacers made of materials of low permeability, the output voltage of the LVDT must be processed with signal conditioners before the estimation of the static sensitivity. It has been shown that the procedure of the calibration is easy and the experimental set-up is simple. Although the proposed measurement method is introduced to a miniature split-Stirling cryocooler, it would be quite practical to apply to other similar applications.

\section{Acknowledgments}

The authors greatly acknowledge the support of the National Science Council under Contract No. NSC820401-D002-033 in Taiwan, ROC. We would also like to thank Professor B.J. Huang, who is responsible for the Thermal and Fluid Control Laboratory of the Department of Mechanical Engineering, National Taiwan University, for his expert advise and helpful discussions.

\section{References}

I1] C.W. de Silva, Control Sensors and Actuators, Prentice Hall, Englewood Cliffs, NJ, 1989.

12] G. Walker, Miniature Refrigerators for Cryogenic Sensors and Cold Electronics, Clarendon Press, Oxford, 1989.

13| E.O. Doebelin, Measurement Systems-Application and Design, McGraw-Hill, New York, 4th edn., 1990.

14| Y. Xiang, Q. Li and F. Guo, Identification of the negative feedback relationship in split cycle free piston Stirling cryocooler system, Cryogenics 30 (September Supplement 1990) 216-220.

[5] T. Zhang, L. Tan, Z. Li and D. Zhang, Experimental investigation on the dynamic pressure distribution in a split piston Stirling cryocooler system, Cryogenics 30 (September Supplement 1990) 221-225.

[6] F. Stolfi and A.K. de Jonge, Stirling cryogenerators with linear drive, Philips Tech. Rev. 42(1) (April 1985) 1-10

17| B.G. Jones and S.R. Scull, Development and preflight qualification testing of a range of cryogenic coolers for applications from $20 \mathrm{~K}$ to $80 \mathrm{~K}$, Cryogenics 32(10) (1992) 850-858.

18| D. Jiles, Introduction to Magnetism and Magnetic Materials, Chapman and Hall, London, 1991

[9] T. Hashimoto, M. Ogawa and R. Li, Recent advance in magnetic regenerator material, Cryogenics 30 (September Supplement 1990) 192-198. 